Meta

Journal des tradlucteurs

Translators' Journal

\title{
Analyse sémantique immanente, référence et traduction
}

\section{Henri Schogt}

Volume 26, numéro 2, juin 1981

URI : https://id.erudit.org/iderudit/004052ar

DOI : https://doi.org/10.7202/004052ar

Aller au sommaire du numéro

Éditeur(s)

Les Presses de l'Université de Montréal

ISSN

0026-0452 (imprimé)

1492-1421 (numérique)

Découvrir la revue

Citer cet article

Schogt, H. (1981). Analyse sémantique immanente, référence et traduction.

Meta, 26(2), 117-122. https://doi.org/10.7202/004052ar d'utilisation que vous pouvez consulter en ligne.

https://apropos.erudit.org/fr/usagers/politique-dutilisation/ 


\title{
Analyse sémantique immanente, référence et traduction*
}

\author{
HenRI SCHOGT
}

La sémantique telle que nous la connaissons aujourd'hui est une branche relativement nouvelle de la linguistique. Qu'on en fasse remonter les origines à Michel Bréal ou à Ferdinand de Saussure, la sémantique moderne a moins de cent ans. Peut-être est-ce dans cet âge tendre qu'il faut chercher l'explication du manque de stabilité et de principes solidement établis, peut-être est-ce la matière même dont la sémantique s'occupe qui lui confêre un caractère insaisissable. Pourtant, si nous voulons savoir quel rôle la sémantique pourra jouer dans le processus de la traduction, il faut d'abord examiner de plus près les traits les plus importants de la sémantique.

La sémantique s'occupe de la signification des éléments linguistiques qui forment l'énoncé, aussi bien que de la signification de l'énoncé lui-même. On peut aller encore plus loin et incorporer les énoncés dans un ensemble plus vaste, le texte ou le discours, mais le principe d'une étude de ce que signifie un élément linguistique formel reste grosso modo intact. Depuis le Cours de linguistique générale de Ferdinand de Saussure (1916), le signe linguistique, unité se composant d'une partie formelle, le signifiant, et d'un contenu idéal, le signifié, occupe une place centrale dans la sémantique européenne. Sans entrer dans le détail de toutes les discussions qui se sont déroulées au sujet du signe linguistique, mentionnons quelques traits importants qui le caractérisent et qui sont assez généralement acceptés :

1) le signe linguistique est arbitraire - le rapport qui existe entre la forme et le contenu idéal repose sur une convention que les membres de la communauté linguistique respectent, mais que ni la forme ni le contenu sémantique ne permettent de deviner aux non initiés;

2) le signe linguistique fonctionne à l'intérieur d'un ensemble de signes par rapport auxquels et grâce auxquels il se délimite;

3) le signe se trouve à un niveau d'abstraction et de généralisation qui permet aux locuteurs d'employer le même signe dans des circonstances variées, l'appliquant à des référents différents. La forme elle aussi n'est pas une phonie unique, mais une abstraction qui est à la base de toutes les phonies acceptables qui se manifestent réellement et qu'on perçoit.

Colloque de Glendon, 1980. 
Saussure n'est pas très clair dans sa terminologie, tantôt il parle du signe comme se composant d'un signifiant et d'un signifié, tantôt on a l'impression que le terme «signe» ne se rapporte qu'au côté signifiant. Il est pourtant certain qu'il faut prendre «signe» dans la première acception quand il est question de la délimitation de chaque signe à l'intérieur d'un système.

C'est lors de l'analyse des unités significatives que les structuralistes de l'école de Genève ainsi que les glossématiciens de Copenhague essayent de suivre le modèle du système phonologique. Henri Frei $(1950$, p. 10) soutient, d'après Saussure, que «dans la langue il n'y a que des différences » et il explique que Saussure "a voulu dire que dans le phénomène langue il n'y a pas d'objet substantiel». Mieux connues que les opinions de Frei, les idées de Louis Hjelmslev (1943) vont dans le même sens. Son ouvrage Omkring Sprogteoriens Grundlaeggelse (Prolégomènes en français, Prolegomena en anglais) ne présente pas de façon intégrale toutes ses idées sur la question - il serait d'ailleurs impossible de les présenter dans une théorie cohérente unique, puisque au cours de sa vie les idées de Hjelmslev ont évolué - mais quand on parle de l'influence de Hjelmslev sur la sémantique, il s'agit toujours des Prolégomènes.

Hjelmslev pose en principe le parallélisme du système des unités de sens, et celui des phonèmes. Poussée jusqu'à ses conséquences extrêmes, cette théorie implique que pour connaître la valeur d'une unité de sens, il faut être au courant de toutes les autres unités aussi. C'est en effet ce qu'on peut déduire du Cours de linguistique, bien que Saussure se soit limité à la discussion de quelques systèmes partiels qui forment le point de départ pour des recherches ultérieures sur ce qu'on appelle les champs sémantiques. Il faut relever quelques problèmes que cette façon de délimiter les unités de sens entraîne et qu'on n'a pas résolus de façon satisfaisante jusqu'à présent.

1) Comme l'inventaire langagier de chaque individu est unique, la valeur des unités qui se délimitent à l'intérieur de cet inventaire est également unique.

2) Si l'on ne tient compte que de la position relationnelle de l'unité par rapport aux autres unités, il est possible — du moins en théorie - de passer sous silence ce à quoi l'unité se réfère dans le monde extralinguistique.

Examinons ces deux problèmes de plus près.

1) La valeur unique de chaque unité de chaque idiolecte semble exclure la communication parfaite. Mais cela ne veut certainement pas dire que toute communication soit impossible. Chaque locuteur sait d'ailleurs que l'interprétation de ce qu'il dit varie d'un interlocuteur à l'autre, même si la base reste presque toujours intacte. Au lieu de partir d'un nombre quasi infini d'idiolectes, il vaut mieux attirer l'attention sur l'élément commun qui est évidemment là, car autrement toute communication serait impossible.

2) Hjelmslev a exclu le côté matériel du phonème, disant que toute notation ou production des unités phonémiques d'un système qui maintient les relations distinctives intactes peut être utilisée. Il serait même de bonne méthode d'utiliser une notation algébrique pour mettre en évidence la dissociation des 
unités phonémiques et leur manifestation sonore. Comme on a affaire à un inventaire limité et fermé, l'exclusion de tout recours à la «substance sonore» lors de l'élaboration du système, bien que peu naturelle, est possible, mais il n'en est pas de même des unités de sens qui appartiennent à un inventaire illimité et ouvert. Hjelmslev fournit des exemples de systèmes partiels, mais ne s'attaque pas à la tâche impossible de structurer le lexique intégral. Remarquons que jusqu'à présent nous avons fait allusion à des unités lexicales, et que pour les unités grammaticales catégorielles la situation ne se présente pas de la même façon. Nous verrons par la suite que les catégories grammaticales offrent peutêtre un point de départ pour entamer la structuration du lexique, mais il faut d'abord voir ce que serait le lexique en tant que système de valeurs sans qu'on fasse appel aux catégories grammaticales.

André Martinet (1975) propose le terme Axiologie pour la discipline qui s'occupe de la valeur des unités significatives du langage (dérivée du mot grec $\alpha \xi i \alpha=$ valeur). Tandis que le système phonologique se compose d'un nombre restreint d'unités dont on peut dégager les traits pertinents à l'aide d'un réseau d'oppositions, le système axiologique compte un nombre quasi infini d'unités, de sorte que l'élaboration des valeurs à l'aide d'une classification et d'un réseau d'oppositions pose des problèmes insurmontables. L'analyse componentielle aboutit à des résultats qui varient d'après le caractère d'un nombre forcément limité d'unités qui servent d'éléments oppositifs. L'exemple bien connu de Katz et Fodor (1963) de bachelor, qu'ils ont décomposé de façon à faire ressortir les éléments pertinents qui caractérisent les différentes acceptions du mot (jeune chevalier, personne qui a obtenu un certain degré universitaire, homme célibataire, jeune phoque mâle sans partenaire pendant la période du rut), montre la complexité des opérations ainsi que l'influence énorme de la réalité extralinguistique sur le choix de ce qui constitue un élément de base.

Comme les unités significatives se réfèrent à quelque chose (Heger (1969) rappelle à ce sujet le aliquid stat pro aliquo de l'antiquité et qui continue à hanter les sémanticiens et les philosophes du langage), un système abstrait de valeurs qui exclut tout recours au référent semble un but difficile à atteindre. Aussi l'axiologie, qui représenterait le même degré d'abstraction que la phonologie, tandis que la sémantique formerait la contrepartie de la phonétique, n'en est-elle encore qu'à ses débuts tandis que la phonologie s'est acquis droit de cité en linguistique depuis longtemps.

Il faut dire que tout en offrant des perspectives théoriques pleines d'attrait, la sémantique immanente ou axiologie n'a pas (encore?) beaucoup à offrir au traducteur. Il est sans doute utile de savoir qu'un certain domaine se divise en trois dans une langue tandis qu'une autre langue connaît deux subdivisions et qu'une troisième langue ne subdivise absolument pas - un peu comme si les labiales $/ \mathrm{p} /, / \mathrm{b} /, / \mathrm{m} / \mathrm{d}$ 'une langue correspondaient à une labiale orale $/ \mathrm{p}-\mathrm{b} /$ et une nasale $/ \mathrm{m} /$, ou à une labiale $/ \mathrm{p}-\mathrm{b}-\mathrm{m} /$ dans d'autres langues. Mais sans l'aide d'un nombre de référents, cette connaissance ne mènera pas très loin, quand on passe d'un système non différencié à un système qui offre le choix de plusieurs unités différentes. Prenons un exemple très simple d'un système partiel. Au 
français ville-village, système à deux termes, s'oppose city-town-village de l'anglais. Les correspondances ne sont pas faciles à établir, et quand un petit patelin de trois cents habitants, chef-lieu de l'île de Block, s'appelle «the town of New Shoreham ", on se demande quels critères permettent de distinguer entre town et village. Les valeurs oppositionnelles de dimension «très grand - moins grand - petit» se combinent avec un nombre d'autres valeurs et c'est en fin de compte l'ensemble des valeurs qui dicte le choix de l'un des termes plutôt que de l'autre. Faut-il en conclure que le terme town a autant d'acceptions qu'il y a de référents? Certainement pas. Dans un livre très intéressant intitulé Inductive Semantics and Syntax, Leo Zawadowski (1975) nous met en garde précisément contre le danger de la fragmentation à partir de la multiplicité des référents. Cette fragmentation s'ajoute à celle qui résulte d'une division de plus en plus poussée qui aboutit à autant de systèmes-idiolectes qu'il y a d'individus qui parlent la langue. Si l'on cherche, au contraire, à dégager les éléments qui permettent la communication au lieu de chercher ce que chaque occurrence a d'unique, et si la base commune des idiolectes est mise en valeur au lieu de souligner ce qui les distingue, on crée une base pour la sémantique qui offre en même temps quelque prise aux traducteurs. Contre cette recherche du général on pourrait formuler l'objection que le traducteur d'un ouvrage littéraire considère l'unique et non pas le général. Pourtant cela n'est que partiellement vrai, car pour trouver ce qui est caractéristique et individuel il faut d'abord avoir établi une base commune à titre de comparaison entre l'écrivain et la communauté linguistique à laquelle il appartient. Il est d'ailleurs difficile de tracer une ligne de démarcation très nette entre la sémantique et la stylistique dans l'opération de la traduction littéraire.

Le problème de la stylistique ne semble pas se poser pour l'école transformationnelle-générativiste qui a marqué profondément la linguistique des années soixante et soixante-dix. Les transformationnistes opèrent à l'aide d'équations qui présupposent l'identité de signification sans faire entrer en considération de différences stylistiques éventuelles. Comme c'est la compétence d'un locuteur idéal qu'on analyse, il est évident que la fragmentation en idiolectes ne menace pas l'homogénéité et la validité générale des résultats. Pourtant les transformationnistes ne sont pas parvenus à bannir la fragmentation dans le domaine des référents. Au lieu de partir de la forme unique et de chercher ce que manger signifie, que ce soit de la soupe, du pain, ou des bonbons acidulés qu'on mange, peu importe, ils attirent l'attention sur le fait que boire commute avec manger quand on a affaire à la soupe, tandis que sucer se trouve être une substitution possible de manger des bonbons acidulés. La décomposition en éléments simples ajoute à la confusion. La phrase très anodine «Dick and Harry went to Cleveland» devient en passant par l'étape «Dick went to Cleveland; Harry went to Cleveland " un message ambigu, puisqu'on ne sait pas s'ils y sont allés ensemble ou séparément. Mais de cette façon tout est ambigu puisque le terme table ne précise pas le nombre de pieds ni la forme du meuble. Ainsi nous retournons dans un certain sens à la linguistique présaussurienne où le principe de l'abstraction et de la généralisation n'avait pas encore été introduit. 
Pour le traducteur, ce débat semble dépourvu d'intérêt, à moins qu'il y ait une langue qui utilise deux verbes différents pour aller ensemble et aller séparément sans avoir le terme générique aller. Roman Jakobson (1959) a déjà attiré l'attention sur la difficulté du choix obligatoire dans la langue d'arrivée (ou langue-cible) quand la langue de départ (ou langue-source) ne fournit pas de renseignements qui permettent de faire ce choix, mais cela ne veut absolument pas dire que le message de la langue qui ne différencie pas soit ambigu. Chaque langue choisit certains traits parmi un nombre de traits possibles pour en faire des traits obligatoirement exprimés tandis que le reste garde un caractère facultatif.

Et cela nous ramène à la structuration des unités significatives d'après leurs traits pertinents. Dans une discussion des idées d'André Martinet sur l'axiologie, Georges Mounin (1979) semble se prononcer en faveur d'une structuration de petits groupes tout en se demandant comment il faudra tracer les limites de ces groupes sans avoir recours à des critères logiques ou conceptuels (voir p. 238-239). Martinet mentionne la possibilité de commencer par les éléments (monèmes) grammaticaux, et d'examiner ensuite les rapports entre ces éléments qu'on a déjà structurés et le lexique. Cette méthode s'utilise à Léningrad où une équipe d'aspectologues, sous la direction de Jurij Sergeevič Maslov et Aleksander Vladimirovič Bondarko (1976), étudie les restrictions lexicales de certaines catégories verbales. Maslov (1978) dirige également des recherches sur la façon dont les langues non slaves comme l'anglais et l'allemand traduisent l'aspect slave. Tout cela n'est qu'un début et ne s'étend qu'à une petite partie du lexique. Pourtant il vaut mieux commencer quelque part de façon modeste et réaliste que de ne pas commencer du tout. Mentionnons aussi les recherches d'Igor Mel'čuk (1974) et son équipe, faites en ce moment à Montréal et qui portent sur les valences et le comportement syntaxique d'unités lexicales.

Même si la sémantique était une discipline solidement établie - ce qu'elle n'est pas - elle ne fournirait que des réponses incomplètes aux questions que lui posent les traducteurs. Dans un article intitulé «Signifié, référent, expérience», Frédéric François (1979) fait ressortir clairement toute la complexité de la communication linguistique et l'impossibilité de formuler des règles précises qui en gouverneraient le fonctionnement toujours et partout. Et nous n'avons même pas parlé des Speech-acts d'Austin et de Searle qui incorporent des couches non cognitives dans leur champ d'investigation. Les sémanticiens n'ont pas de formules simples à offrir aux traducteurs. Ceux-ci profiteront pourtant certainement des résultats que la sémantique a obtenus dans des recherches qui visent à structurer au moins une partie des unités de sens, notamment les monèmes grammaticaux. Il ne faut pas s'attendre toutefois comme le dit le sémanticien anglais F. R. Palmer à «a massive breakthrough». Il continue - et ce sont les dernières phrases de son livre (1976) aussi bien que de mon introduction d'aujourd'hui: «The complexity of semantics is merely one aspect of the complexity of human language. What we can say will be imprecise and often controversial. There are no easy answers. » 


\section{BIBLIOGRAPHIE}

BONDARKO, Aleksander Vladimirovič, «Kategorial'nye i nekategorial'nye značenija $\vee$ grammatike » (Significations catégorielles et non catégorielles en grammaire), in Principy i metody semantičeskix issledovanij (Principes et méthodes de recherches sémantiques), Moscou, Nauka, 1976, p. 180-202.

FRANÇOIS, Frédéric, «Signifié, référent, expérience», in Linguistique fonctionnelle, débats et perspectives, (éd. Mortéza Mahmoudian), Paris, P.U.F., 1979, p. 241-259.

FREI, Henri, «Saussure contre Saussure? », Cahiers Ferdinand de Saussure IX (1950), p. 7-28.

HEGER, Klaus, «L'analyse sémantique du signe linguistique», Langue française I, 4 (1969), p. 44-66.

HJELMSLEV, Louis, Omkring sprogteoriens grundlaeggelse, Copenhague, B. Lunos bogtrykkeri, 1943. trad. angl. J. Whitfield, Prolegomena to a Theory of Language, en suppl. de International Journal of American Linguistics, XIX, 1 (1953); revised edition Madison, Wisc., U. of Wisconsin P., 1961, 1969². trad. franc. A.-M. Léonard, Prolégomènes à une théorie du langage, Paris, Ed. de Minuit, 1968.

JAKOBSON, Roman, "On linguistic aspects of translation», in On Translation, (réd. Reuben A. Brower), Cambridge, Mass., Harvard U.P., 1959, p. 232-239.

KATZ, Jerrold and Jerry FODOR, "The structure of a semantic theory», Language XXXIX (1963), p. $170-210$.

MARTINET, André, «Sémantique et axiologie», Revue roumaine de linguistique XX (1975), p. 539-542.

MASLOV, Jurij Sergeevič (réd.), Voprosy sopostavitel'noj aspektologii (Problèmes d'aspectologie comparée), Leningrad, Izd. Len. Univ., 1978.

MEL'ČUK, Igor, «Esquisse d'un modèle linguistique du type 'Sens $\longleftrightarrow$ Texte'», in Problèmes actuels en psycholinguistique, Colloques intern. du CNRS, $\mathrm{n}^{\circ}$ 206, Paris, 1974.

MOUNIN, Georges, «L'axiologie d'André Martinet», in Linguistique fonctionnelle, débats et perspectives (réd. Mortéza Mahmoudian), Paris, P.U.F., p. 229-239.

PALMER, Frank R., Semantics, Cambridge-Londres-New York-Melbourne, Cambridge U.P., 1976.

SAUSSURE, Ferdinand de, Cours de linguistique générale, Paris-Lausanne, Payot, 1916.

ZAWADOWSKI, Leo, Inductive Semantics and Syntax, La Haye-Paris, Mouton, 1975. 